\title{
Power Scaling Limits in High Power Fiber Amplifiers due to Transverse Mode Instability, Thermal Lensing and Fiber Mechanical Reliability
}

\author{
Michalis N. Zervas *a,b \\ ${ }^{\mathrm{a}}$ Optoelectronics Research Centre, University of Southampton, SO17 1BJ, UK; \\ ${ }^{b}$ Advanced Laser Lab, ORC/SPI Lasers, University of Southampton, SO17 1BJ, Southampton, UK
}

\begin{abstract}
We introduced a simple formula providing the mode-field diameter shrinkage, due to heat load in fiber amplifiers, and used it to compare the traditional thermal-lensing power limit $\left(\mathrm{P}_{\mathrm{TL}}\right)$ to a newly developed transverse-mode instability (TMI) power limit $\left(\mathrm{P}_{\mathrm{TMI}}\right)$, giving a fixed ratio of $\mathrm{P}_{\mathrm{TMI}} / \mathrm{P}_{\mathrm{TL}} \approx 0.6$, in very good agreement with experiment. Using a failurein-time analysis we also introduced a new power limiting factor due to mechanical reliability of bent fibers. For diode (tandem) pumping power limits of $28 \mathrm{~kW}(52 \mathrm{~kW})$ are predicted. Setting a practical limit of maximum core diameter to $\sim 35 \mu \mathrm{m}$, the limits reduce to $15 \mathrm{~kW}(25 \mathrm{~kW})$.
\end{abstract}

Keywords: high power fiber lasers, high power fiber amplifiers, transverse mode instability, thermal lensing, mechanical reliability, power scalability

\section{INTRODUCTION}

High power fiber lasers (HPFLs) have shown record single-moded diffraction-limited output powers in the range of 10$20 \mathrm{~kW}$ [1]. Output power limits in cladding-pumped HPFLs are shown to be set by the pump brightness, optical nonlinearities (such as stimulated Raman (SRS) or Brillouin (SBS) scattering), optical damage, glass rapture or melting and thermal lensing (TL) [2,3]. The TL power threshold has been set arbitrarily to a level that results in mode field diameter (MFD) $\omega_{\mathrm{TL}} \approx 0.7-0.8 \omega_{0}$, where is $\omega_{0}$ the initial ("cold") MFD [2]. Therefore, its impact on power scalability is somewhat artificial. It has been shown that in diode-pumped HPFLs the output power limit is set to a value of $\sim 37 \mathrm{~kW}$ [3] by the combination of SRS and TL. In the case of in-band pumping the predicted power limit is increased to $97 \mathrm{~kW}$ [4].

More recently, transverse mode instability (TMI) has been identified as a severe limiting effect and its impact on power scaling has been considered [5,6]. In these studies, different TMI power threshold formulae were considered giving different power limits. In [5] the TMI threshold is considered to be reached at a fixed average heat load of $\mathrm{Q}_{0}=34 \mathrm{~W} / \mathrm{m}$, independent of core diameter [7], and predicts a diode-pumped power limit of 70kW, defined though by SRS and TL. In [6] the TMI threshold scales inversely with core area and the diode-pumped power limit is $\sim 28 \mathrm{~kW}$. Moreover, the substantially increased power limits were achieved with fiber length/core diameters of $\sim 80 \mathrm{~m} / 220 \mu \mathrm{m}$ [4] and $\sim 120 \mathrm{~m} / 260 \mu \mathrm{m}$ [5]. Following these results, it becomes apparent that efforts should be made to establish the relation between TL and TMI thresholds, as well as, consider additional constraints imposed by the predicted long fiber lengths and large core/cladding diameters.

\section{THERMAL LENSING \& TMI COMPARISON}

In this work, we first adapt a recently derived simple TMI power threshold formula [8,9], namely: 


$$
P_{T M I}=\frac{\kappa U_{11}^{2}\left(U_{11}^{2}-U_{01}^{2}\right)}{2 \pi n_{\text {eff }}\left[\eta_{\text {heat }} /\left(\eta_{\text {laser }} L\right)\right](d n / d T)}\left(\frac{\lambda_{0}}{d_{0}}\right)^{2}
$$

in terms of laser efficiency ( $\left.\eta_{\text {laser }}\right)$, heat generation coefficient ( $\left.\eta_{\text {heat }}\right)$ and amplifier length $(L)$. The other parameters are as follows: $U_{11}$ and $U_{01}$ are the transverse wavenumbers of the perturbation $\left(L P_{11}\right.$ mode) and fundamental $L P_{01}$ mode (FM), respectively, $\kappa$ is the silica thermal conductivity, $(d n / d T)$ is the thermo-optic coefficient and $n_{\text {eff }}$ is the FM effective index. $\lambda_{0}$ is the signal wavelength and $d_{0}$ is the core diameter. For $P \geq P_{\text {TMI }}$ small transverse amplitude and/or phase perturbations grow exponentially, scatter light into to HOMs and lead to TMI. For $P<P_{\text {TMI }}$ they die out and the FM amplification is stable.

Thermal lensing in optical fibers has also been described in terms MFD reduction and a threshold power is derived [2], namely:

$$
P_{T L}=\frac{2 \pi \kappa}{\left[\eta_{\text {heat }} /\left(\eta_{\text {laser }} L\right)\right](d n / d T)}\left(\frac{\lambda_{0}}{d_{0}}\right)^{2}
$$

The TL power threshold has been set arbitrarily to a level that results in MFD $\omega_{\mathrm{TL}} \approx 0.7-0.8 \omega_{0}$, where is $\omega_{0}$ the initial ("cold") MFD. Comparing Eqn. (1) and (2), we realize that $\mathrm{P}_{\text {TMI }}$ and PTL both show a $\left(\lambda_{0} / \mathrm{d}_{0}\right)^{2}$ dependence. Fig. 1(a) compares $\mathrm{P}_{\mathrm{TMI}}$ and $\mathrm{P}_{\mathrm{TL}}$ power thresholds (see inset), showing a ratio $\mathrm{P}_{\mathrm{TMI}} / \mathrm{P}_{\mathrm{TL}} \approx 0.6$. It is shown that TMI occurs before the TL limit is reached. It should also be stressed that TMI results in unstable laser output and constitutes a real power limit. Thermal lensing, on the other hand, results in MFD reduction and only affects the laser performance indirectly, mainly due to increased nonlinearities.

We have also compared the experimentally obtained MFD reduction (limited by TMI) with extracted power (open circles) [10] and compared it with a simplified formula [11], which gives the MFD in quasi-parabolic RI profiles (solid lines) due to amplifier heat load $\left(\mathrm{Q}_{0}\right)$ (see formulae in Fig.1(b) inset). Fig. 1(b) plots the results for three $1.2 \mathrm{~m}$ long

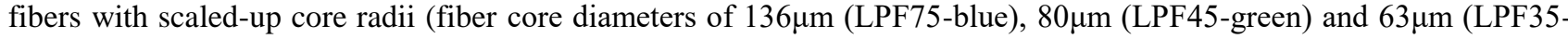
red)), showing a very good agreement. In addition, we have marked the experimentally observed $\mathrm{P}_{\text {TMI }}$ (blue arrows) and the expected $\mathrm{P}_{\mathrm{TL}}$ limit, for which $\omega_{\mathrm{TL}} \approx 0.7 \omega_{0}$ (red arrows). In all cases, the ratio $\mathrm{P}_{\mathrm{TMI}} / \mathrm{P}_{\mathrm{TL}} \approx 0.65$, in very good agreement with the results in Fig. 1(a). It is observed that TMI sets in well before the conventional TL limit is reached, following closely a $\left(1 / \mathrm{d}_{0}\right)^{2}$ dependence in very good agreement with our theory. It should be noted that use of the alternative TMI power threshold [7], which in this case takes the simplified form $\mathrm{P}_{\text {TMI2 }}=34\left(\eta_{\text {laser }} / \eta_{\text {heat }}\right) \mathrm{L}$, would have resulted in a TMI threshold of $\sim 105 \mathrm{~W}$, independent of core diameter.
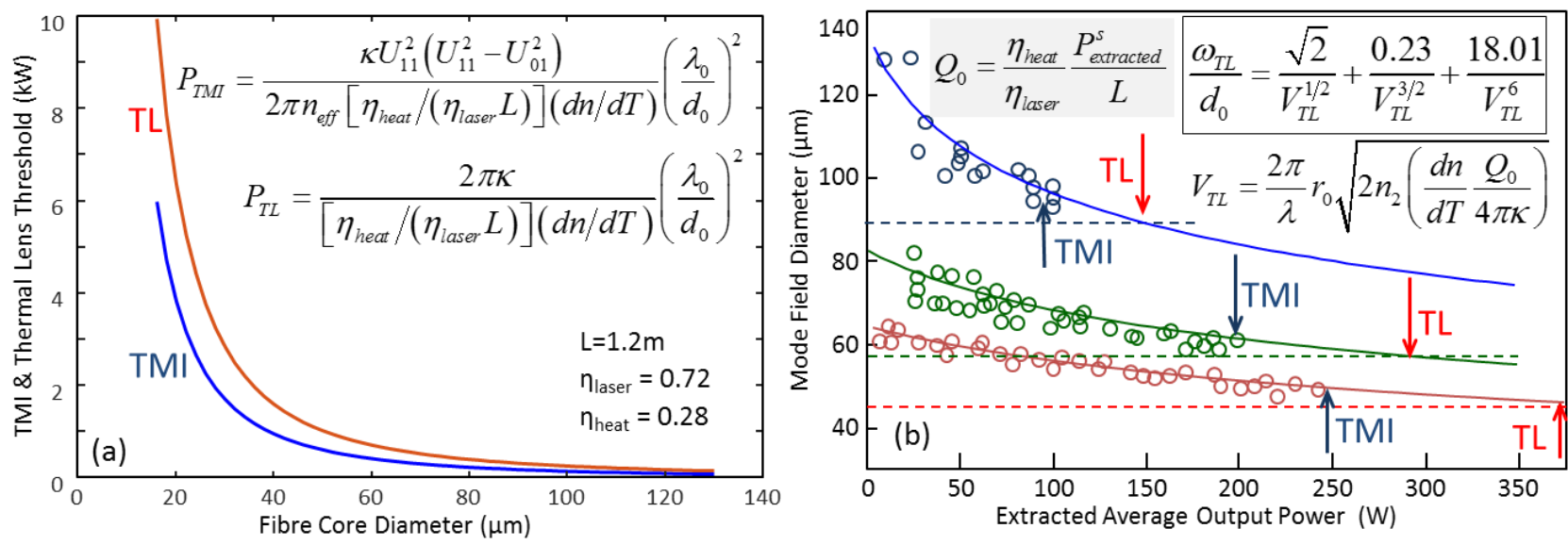

Fig.1: (a) TMI\&TL power thresholds, (b) MFD shrinkage with extracted power (exp [10] vs theory) 


\section{MECHANICAL RELIABILITY}

In all previous power scaling investigations, a fixed core pump absorption ( $\mathrm{Yb}^{3+}$ concentration) was considered and as a result large core diameters were accompanied by large cladding diameters $(>1 \mathrm{~mm})$ and long fiber lengths $(>>10 \mathrm{~m})$ [2-6]. Given that most practical industrial or directed energy HPFL systems should preferably have minimum footprint, the additional constraints of mechanical reliability of bent fibers should be considered. Fig. 2 shows the calculated failuresin-time (FIT) as a function of fiber bending diameter $\left(D_{\text {bend }}\right)$, for different fiber cladding diameters. The fiber is assumed to have undergone minimum proof testing and the failure time is 20years [12,13]. Assuming a maximum bending diameter of $1 \mathrm{~m}$, the maximum allowable cladding diameter is $\sim 600 \mu \mathrm{m}$. This limit will be used in the subsequent calculations.

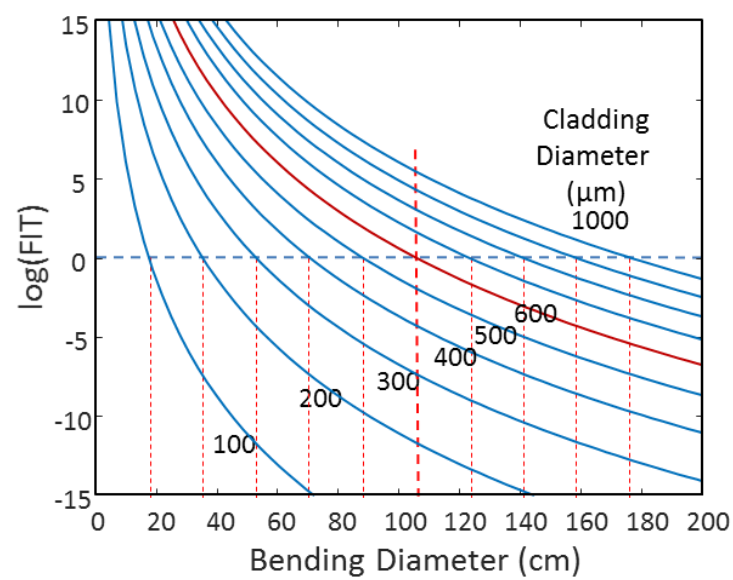

Fig.2: Failures-in-Time (FIT) versus bending diameter $\left(D_{\text {bend }}\right)$

\section{POWER SCALING}

We now investigate the power scaling limits in HPFLs taking into account the newly developed TMI power threshold and the constraints due to fiber mechanical reliability, in addition to the previously considered effects. The other power limiting effects and fiber parameters are similar to the ones used in the literature [2][3].

Fig. 3(a) plots the power scaling limits due to pump brightness, SRS and TMI, with parameters similar to Ref. [2] $\left(\mathrm{B}_{\mathrm{p}}=0.02 \mathrm{~W} / \mu \mathrm{m}^{2} / \mathrm{sr} ; \eta_{\text {laser }}=0.84 ; \eta_{\text {heat }}=0.10(976 \mathrm{~nm}\right.$ diode pumping $)$ ). Due to lower TMI threshold, the maximum power is reduced from $34 \mathrm{~kW}$ to $\sim 28 \mathrm{~kW}$. However, when we consider the additional mechanical reliability constraint the maximum power reduces to $\sim 3 \mathrm{~kW}$. Increasing the pump brightness $B_{p}=0.2 \mathrm{~W} / \mu \mathrm{m}^{2} / \mathrm{sr}$ reduces the fiber length and core diameter requirements and results in a maximum power of $\sim 28 \mathrm{~kW}$ (see Fig. 3(b)). Increasing the amplifier gain to $23 \mathrm{~dB}$, on the other hand, increases the SRS/TMI-limited power to $42.5 \mathrm{~kW}$, but when we impose the mechanical reliability limit the maximum power reduces to $\sim 15 \mathrm{~kW}$. Finally, in the case of tandem pumping $\left(\mathrm{B}_{\mathrm{p}}=0.3 \mathrm{~W} / \mu \mathrm{m}^{2} / \mathrm{sr} ; \eta_{\text {laser }}=0.90 ; \eta_{\text {heat }}=\right.$ $0.03 \lambda_{\mathrm{p}}=1018 \mathrm{~nm}$ ), the maximum power is increased to $\sim 52 \mathrm{~kW}$ for a core diameter of $\sim 53 \mu \mathrm{m}$. However, for a more practical core diameter of $\sim 35 \mu \mathrm{m}$ the maximum achievable power reduces to $\sim 20 \mathrm{~kW}$. 

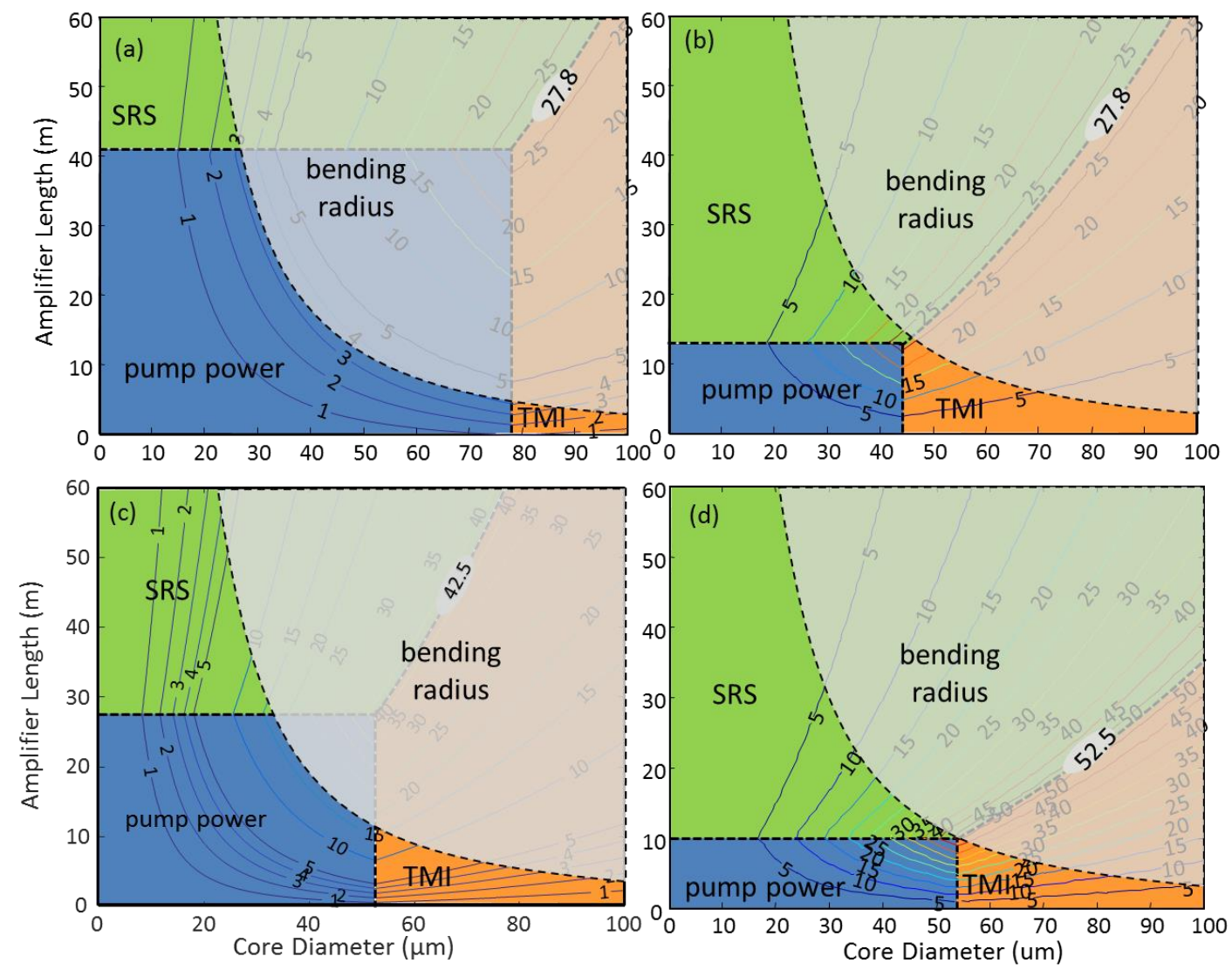

Fig. 3: Power limits in HPFLs (fixed core pump absorption: 250dB/m)

\section{SUMMARY}

In summary, we have introduced a simple formula providing the MFD shrinkage due to heat load and used it to compare traditional $\mathrm{P}_{\mathrm{TL}}$ formula [2] to the newly developed $\mathrm{P}_{\mathrm{TMI}}$ one [8,9], giving a fixed ratio of $\mathrm{P}_{\mathrm{TMI}} / \mathrm{P}_{\mathrm{TL}} \approx 0.6$, in very good agreement with experimental results. This demonstrates that TMI occurs before the traditional TL limit is reached. Using a FIT analysis we have also introduced a maximum allowable cladding diameter of $\sim 600 \mu \mathrm{m}$ as a new power limiting factor related to the mechanical reliability of bent fibers. We used these new findings to investigate the power scaling in HPFLs. With increased brightness diode pumping a power limit of $28 \mathrm{~kW}$ is predicted. For tandem pumping, on the other hand, the power limit increases to $\sim 52 \mathrm{~kW}$. If we set a practical limit of the maximum core diameter to $\sim 35 \mu \mathrm{m}$, the limits reduce to $15 \mathrm{~kW}$ and $25 \mathrm{~kW}$, respectively.

\section{Acknowledgements}

This project was supported by the Royal Academy of Engineering under the Research Chairs and Senior Research Fellowships Scheme.

\section{REFERENCES}

[1] Zervas, M. N. and Codemard, C. A., "High Power Fiber Lasers: A Review," J. Select. Top. Quantum Electron., 20, 0904123 (2014). 
[2] Dawson, J. W., Messerly, M. J., Beach, R. J., Shverdin, M. Y., Stappaerts, E. A., Sridharan, A. K., Pax, P. H., Heebner, J. E., Siders, C. W. and Barty, C. P. J., "Analysis of the scalability of diffraction-limited fiber lasers and amplifiers to high average power," Opt. Express 16, 13240 (2008).

[3] Dawson, J. W., Messerly, M. J., Heebner, M. J., Pax, P. H., Sridharan, A. K., Bullington, A. L., Beach, R. J., Siders, C. W., Barty, C. P. J. and Dubinskii, M., "Power scaling analysis of fiber lasers and amplifiers based on nonsilica materials," Proc. SPIE 7686, 768611 (2010).

[4] Ke, W. W., Wang, X. J., Bao, X. F. and Shu, X. J., "Thermally induced mode distortion and its limit to power scaling of fiber lasers," Opt. Express 21, 14272 (2013).

[5] Otto, H. J., Jauregui, C., Limpert, J. and Tünnermann, A., "Average power limit of Ytterbium-doped fiber-laser systems with nearly diffraction-limited beam quality," Proc. SPIE 9728, 97280E (2016).

[6] Zervas, M. N., "Power Scalability in High Power Fibre Amplifiers," CLEO/Europe-EQEC, paper CJ-6.1 (2017).

[7] Jauregui, C., Otto, H. J., Breitkopf, S., Limpert, J. and Tünnermann, A., "Optimizing high-power Yb-doped fiber amplifier systems in the presence of transverse mode instabilities," Opt. Express 24, 7879 (2016).

[8] Zervas, M. N., "TMI Threshold in High Power Fiber Amplifiers," paper SoW2H.2, OSA Advanced Photonics Congress (2016).

[9] Zervas, M. N., “Transverse Mode Instability Analysis in Fibre Amplifiers,” Proc. SPIE 10083, 100830M (2017).

[10] Jansen, F., Stutzki, F., Otto, H. J., Eidam, T., Liem, A., Jauregui, C., Limpert, J. and Tünnermann, A., "Thermally induced waveguide changes in active fibers," Opt. Express 20, 3997 (2012).

[11] Marcuse, D., "Loss analysis of single-mode fiber splices," Bell Syst. Techn. J. 56, 703 (1977).

[12] Mitsunaga, Y., Katsuyama, Y., Kobayashi, H. and Ishida, Y., "Failure prediction for long length optical fiber based on proof testing," J. Appl. Phys. 53, 4847 (1982).

[13] Yamada, M., Tsujikawa, K., Ma, L., Ichii, K., Matsuo, S., Hanzawa, N. and Ono H., “Optical Fiber Amplifier Employing a Bundle of Reduced Cladding Erbium-Doped Fibers,” IEEE Photon. Techn. Lett. 24, 1910 (2012). 